\title{
Determination of Location and Amount of Series Compensation to Increase Power Transfer Capability
}

\author{
Rajesh Rajaraman, Fernando Alvarado*, Arthur Maniaci, Robert Camfield, and Sasan Jalali** \\ Laurits R. Christensen Associates, Inc. \\ Economics and Engineering Consultants \\ 4610 University Avenue, Madison, WI 53705-2164 \\ Phone: (608) 231-2266; Fax: (608) 231-1365; email: rajesh@lrca.com
}

*Department of Electrical and Computer Engineering,

University of Wisconsin-Madison, Madison, WI 53706

** Siemens Energy and Automation

Atlanta, GA

\begin{abstract}
This paper discusses the merits of an analytical method for determining the location and amount of series compensation to increase the steady state power transfer capability in the power system based primarily on cascading line overload considerations. Steady state transmission limits define the boundaries of a power system operating point. This paper presents tools to identify these boundaries and analytically estimates the change in the boundary limits due to the application of series and shunt compensation. The approach identifies critical lines that can initiate cascading line outages and identifies the optimal location and amount for series compensation. The methodology can also be used to select sites for devices that can extend the availability of power in flowgates.
\end{abstract}

\section{Introduction}

This paper describes applications of an analytical approach to the problem of sizing and locating series and shunt compensation. The paper is the result of collaboration between the Power Markets and Resource Management business unit (PM\&RM) of the Electric Power Research Institute (EPRI), Christensen Associates (LRCA) and Siemens.

Understanding the limitations of a power transmission network is fundamental to both integrated systems and competitive markets for electric energy. Patterns of generation that result in heavy flows tend to incur greater losses, ultimately making certain generation patterns economically undesirable. Transmission capability constraints rule out certain combinations of supply and demand, under the threat of likely or potential power outages of varying severity. Ignoring either characteristic

PE-672-PWRS-1-07-1997 A paper recommended and approved by the IEEE Power System Analysis, Computing and Economics Committee of the IEEE Power Engineering Society for publication in the IEEE Transactions on Power Systems. Manuscript submitted January 2, 1997; made available for printing August 19, 1997. of the power systems in network planning is likely to lead to higher system costs and reduced reliability. This paper addresses transmission capability limits and concerns itself primarily with planning decisions.

Network capabilities, often described as Available Transmission Capability (ATC) [1], can be defined in several says, including: (a) the notion of "flowgates," or corridors within the power system that under certain conditions become limiting [2]; (b) the notion of the "Operational Limit Boundary" (OLB), which defines in demand space all combinations of consumption that are compatible with a given state of the transmission network [3]; and (c) the notion of outage costs, which estimates the expected value of the cost of the potential outages incurred by network users that may result as a consequence of operating at a given demand and generation pattern [4]. These three notions are tightly interconnected, and the tools developed for any one of them are often of direct applicability to the other two concepts.

Capability limits can be attributed to overloads on lines or transformers, low voltage or near voltage collapse states, or dynamic instability that evolves from load and generation patterns under some conditions in the power grid. Of greatest concern are overloads, particularly those leading to cascading line outages. The widespread outages in the Western System of the US during July and August 1996 were ultimately a result of cascading line outages.

Although cascading overloads have been a concern for many years, the formalization of an analytical approach to quantify margins to cascading conditions, including sensitivities of various system actions, has not been fully accomplished to date. However, such approach is of interest to all participants in the electric services industry, particularly system planners. Compensation technologies 
are costly; planners and vendors of power technologies wish to have the analytical capability and tools to site compensation devices where the net benefits to the system are greatest. Our approach follows the pattern of a similar effort by Dobson and his fellow researchers ([5], [6], [7], and [8]) on the quantification of margins to voltage collapse conditions. Indeed, the efforts of this paper may be viewed as an application of the work of Dobson. In addition, Van Cutsem, e.g., [9] has inspired the development of the present approach.

Analyses of networks and available transmission capability often assume that the facilities that comprise the grid are known, and remain fixed over time. This is of course a poor representation of network facilities, which assume a long term time dimension. Indeed, networks evolve as they respond to changing states of demand and supply, both of which are specific to location. Networks are continually redesigned and modified, subject to cost, timing and siting considerations. One of the most effective means to modify the grid is series compensation devices. These devices alter the series impedance of lines and transformers, thus altering the flows in the grid, sometimes in dramatic ways [10].

The paper proceeds by reviewing Operational Limit Boundary (OLB) and power transfer capability in Sections 2 and 3. The formula to calculate of the effect of both generation redispatch and series device placement is provided and interpreted in Section 4 (detailed derivations are given in the appendix). Section 5 illustrates the approach and verifies the formula. Along with the implications of the present work on the future of series compensation devices, the implications for power transfer capability are briefly discussed in Section 6 .

\section{The Operational Limit Boundary}

The transmission network is limited in the amount of power that can be delivered to loads in the system. The boundary of all feasible load combinations is the Operation Limit Boundary (OLB). Cascading line overloads arise when:

1. A transmission line or a set of lines reaches a flow limit, and

2. Generator redispatch fails to relieve the overload(s), and

3. Opening the overloaded line(s) results in further overloads in other lines. Opening those lines, in turn, further overloads other lines, leading to cascading overloads, and may ultimately give rise to forced outage of loads.
The OLB can be conveniently viewed in a multidimensional space. Each axis in this space represents either a contract transaction or a load at a bus or zone. In Figure 1, the horizontal and vertical axes represent either the electric power demand of two loads $A$ and $B$, or two bulk power transactions.

In general, the "dimension" of this space is much greater than two; there are as many dimensions as there are independent transactions or loads. If an operating point lies within the OLB, it can be physically realized, and is said to be feasible. Operating points projected to lie outside the OLB result in cascading line overloads and must be curtailed to positions within the OLB. Hence the OLB represents the (steady-state) frontier of power transfer capability.

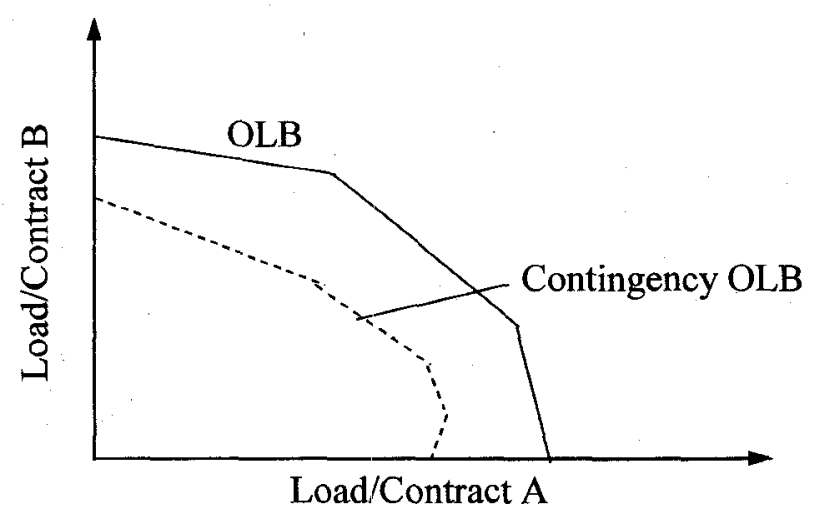

Fig. 1 - Operation Limit Boundary (OLB)

The OLB also depends upon exogenous factors such as the status of the transmission network and the availability of generation units. These exogenous factors affect the position of the OLB. The OLB is also affected by series compensation which could expand the frontier. Estimating the entire OLB is unnecessary; the next section shows that it is sufficient to obtain information about a few points on the OLB.

\section{Power Transfer Capability}

Compensation technologies are evaluated by transmission planners in terms of both costs and benefits over the planning horizon. Evaluation should take account of uncertain of various supply and demand states, including:

1. Load growth outlook,

2. Generation expansion, and

3. Various transmission planning scenarios and contingencies.

For each scenario of load growth, generation, and transmission plan, the power transfer capability is defined 
as the margin from a base case operating point to the $O L B$, measured along the load growth trajectory (Figure 2). Typically, the margin would be in MW, but it could also be a combination of MW and MVAR. Adding series compensation results in a change in the power transfer capability.

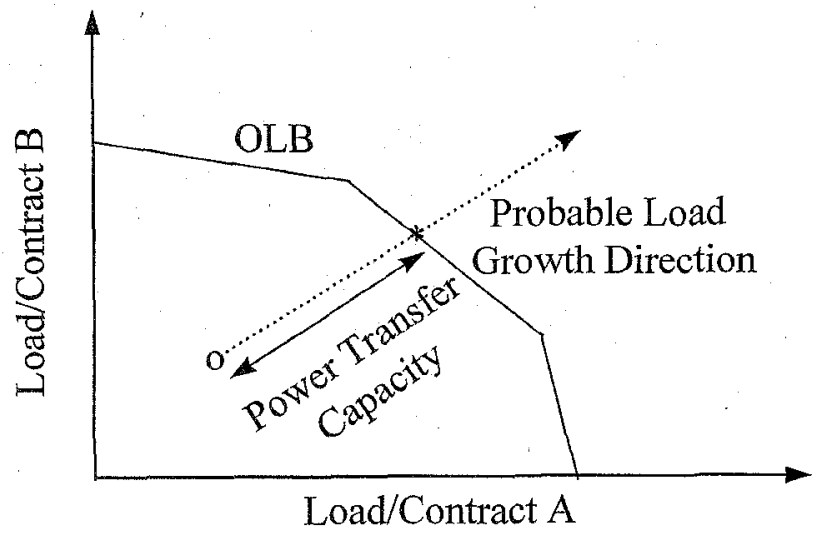

Fig. 2 - Transfer Capability Along Load Direction.

For each possible state of the world scenario, the change in power transfer capability due to series compensation can be converted into economic terms (\$) for use within a cost-benefit framework (CBF). CBF embraces an objective function; least-cost planning principle state that total value to the participants (economic agents) of an electric market are maximized when the expected value of the discounted marginal benefit and costs are equivalent. That is, all grid expansion opportunities are exhausted. The sheer number of alternatives can be very large; indeed, the objective may be to maximize power transfer capability subject to the constraint that the costs of compensation be less than the costs incurred using alternative methods such as demand side management and load curtailment programs. However the objective function is defined, the minimum information to successfully conduct a compensation study is: (a) the power transfer capability, and (b) the change in power transfer capability due to compensation. The analysis to attain this information is required for each combination of load growth, generation and transmission planning scenario.

\section{Series Compensation Sensitivity Formulas}

First, consider a set of scenarios including load growth, generation expansion and transmission plans, along with accompanying contingency events and their likelihood. The transfer capability for each scenario can be determined as follows:
1. Start from a base case operating point (point o in Figure 2)

2. Solve continuation load flows by increasing load amounts in the forecast direction and assume that the appropriate generation policies are in effect.

3. If, during the continuation load flow, a line exceeds its flow limit, attempt generation redispatch.

4. If the generation redispatch is unable to relieve the flow limit, (or if the cost of doing so exceeds a predetermined threshold), open the transmission line. If this leads to further overloads, go to Step 3. In case of cascading overloads, go to Step 5. If all overloads are resolved, go to Step 2.

5. The OLB (point $*$ in Figure 2) has been reached when cascading overloads occur. Identify the critical transmission line whose flow limit causes the start of the cascading overloads.

6. The power transfer capacity is the difference of the total system loads at points * and $\mathbf{o}$.

We illustrate this procedure in Table 1 for a hypothetical case when no redispatch is done. In Table 1 , a continuation load flow is done for various load levels for a hypothetical system. The point of cascading overloads occurs when the load level is $1543 \mathrm{MW}$ and the critical transmission line that sets off the cascading overloads is $35-52$.

Table 1 - Sequence of Events Leading to Cascading Overloads. Line 35-52 is the critical line.

\begin{tabular}{|l|l|}
\hline $\begin{array}{c}\text { Load } \\
\text { (MW) }\end{array}$ & Remarks \\
\hline $\mathbf{1 0 0 0}$ & OK \\
\hline $\mathbf{1 2 0 0}$ & OK \\
\hline $\mathbf{1 2 6 2}$ & Overload line 19-30 \\
\hline $\mathbf{1 2 6 2}$ & Open line 19-30; OK \\
\hline $\mathbf{1 5 0 0}$ & OK \\
\hline $\mathbf{1 5 4 3}$ & Overload line 35-52 \\
\hline $\mathbf{1 5 4 3}$ & Open 35-52; Line 18-78 overloaded \\
\hline $\mathbf{1 5 4 3}$ & Open 18-78; Line 32-60 overloaded \\
\hline $\mathbf{1 5 4 3}$ & Open 32-60; Cascading overloads! \\
\hline
\end{tabular}

When generator redispatch is used as a means to relieve some line overloads (in conjunction with the option of opening the overloaded line), the transmission planner must judge which redispatch is "too costly" -i.e., higher energy costs. In a competitive power market, as defined in ISO protocols, redispatch policies could be outside of the transmission planner's purview. Thus, the planner may have to analyze scenarios of likely redispatch actions, given expected power market arrangements. Figure 3 shows that, as the maximum allowed redispatch 
costs vary, it is possible to obtain different measures of power transfer capability and different locations of the critical lines. This will affect the location of series compensation devices.

\section{Figure 3 - Power Transfer Capacity Depends on Redispatch Policy}

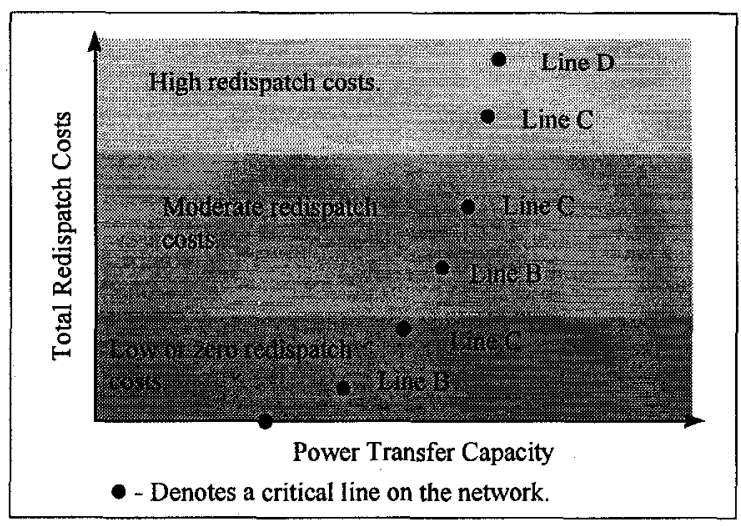

As a practical matter, several critical lines may be identified for each load growth, generation expansion and transmission scenario. The following analysis steps can be repeated for each critical line. Let one such critical transmission line causing the cascading overloads be between buses $m$ and $n$ (with the flow direction from $m$ to $n$ ). Now suppose series compensation is added in the line between buses $r$ and $s$. Assume that flow limits are given in MW (Appendix A gives the corresponding formula for the case when the flow limits are expressed in MVA), Appendix A shows that the change in power transfer capacity $\Delta \alpha$ in response to an addition of $\Delta t \%$ series compensation in line between $r$ and $s$ with admittance $\mathrm{y}_{\mathrm{rs}}=\mathrm{g}_{\mathrm{rs}}+\mathrm{jb} \mathrm{r}_{\mathrm{rs}}$ can be estimated by:

$$
\Delta \alpha=\Lambda_{1 \mathrm{G}} \frac{\partial \mathrm{b}_{\mathrm{rs}}}{\partial \mathrm{t}} \Delta \mathrm{t}+\Lambda_{2 \mathrm{G}} \frac{\partial \mathrm{g}_{\mathrm{rs}}}{\partial \mathrm{t}} \Delta \mathrm{t}
$$

where $\Lambda_{1 \mathrm{G}}$ and $\Lambda_{2 \mathrm{G}}$ are the sensitivities of the power transfer capacity with respect to $b_{\mathrm{rs}}$ and $\mathrm{g}_{\mathrm{rs}}$ respectively. The subscript $G$ indicates that $\Lambda_{1 G}$ and $\Lambda_{2 G}$ depend on the generation dispatch policies. Appendix $A$ gives explicit procedures for computing the quantities $\Lambda_{1 \mathrm{G}}, \Lambda_{2 \mathrm{G}}$ in terms of the generation dispatch policy, the bus voltages, and angles of the system at its point of cascading overloads (point * on the OLB). The terms $\frac{\partial \mathrm{b}_{\mathrm{rs}}}{\partial \mathrm{t}}, \frac{\partial \mathrm{g}_{\mathrm{rs}}}{\partial \mathrm{t}}$ are computed in a simple way from equation (A7).

Once the point $\left(^{*}\right)$ of cascading overloads is found, the effect of series compensation in any transmission line on the power transfer capability may be analytically estimated from equation (1). Besides saving significantly on computational time by not re-computing the power transfer capacity for each possible case of series compensation, equation (1) also indicates the locations where the compensation could be performed. Another important byproduct of equation (1) is that it indicates the change in power transfer capability with respect to various generation dispatch policies. Thus, it also indicates where new generation capacity may be "advantageously" sited.

\section{Results and Discussion}

This section illustrates the application of formula (1) on a simple test system (Figure 4) and compares the results to a numerical simulation of the network. The primary advantage of the analytical method is that it requires just one point-of-collapse study for any critical line examined. Furthermore, our approach is equally valid for very large systems. Indeed, it is currently being employed in a more detailed fashion for a large Eastern European power system [1].

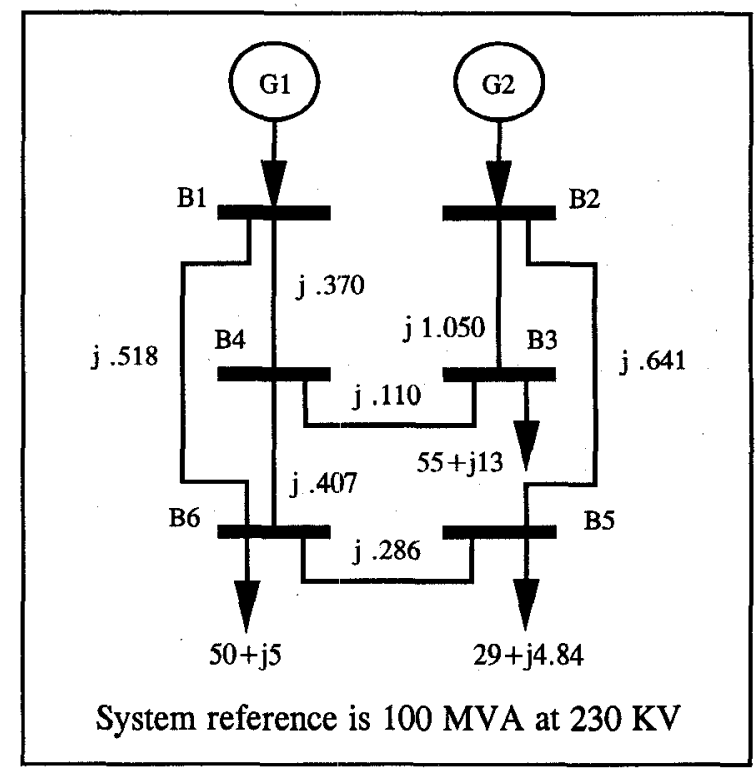

Fig. 4

A load growth scenario was assumed: Loads at buses 3,5 , and 6 grow at $5 \%, 3 \%$, and $2 \%$ respectively. A merit order dispatch was assumed: Generator 1 was first dispatched until it reached a limit of $60 \mathrm{MW}$, then Generator 2 was dispatched. The point of cascading overloads was obtained using LRCA's program PFLOW [11]. At this point, the critical transmission line causing the overloads was identified as line 1-4, and the system Jacobian, bus voltages and angles were obtained. Equation (1) now estimates the change in power transfer capacity with respect to different levels and location of series compensation. To verify this, we attempted series compensation in every line (excepting the transformer line 3-4) and for various compensation levels, and computed the actual change in transfer capacity as a result of the compensation. 
The test results are shown in Figure 5 and Table 2. From Figure 5, we observe that power transfer capability can be increased or decreased depending on the line selected for compensation. In most cases, the deviation between the actual and simulated results is quite small, with the exception of line 2 (from generator bus 1 to load bus 6).

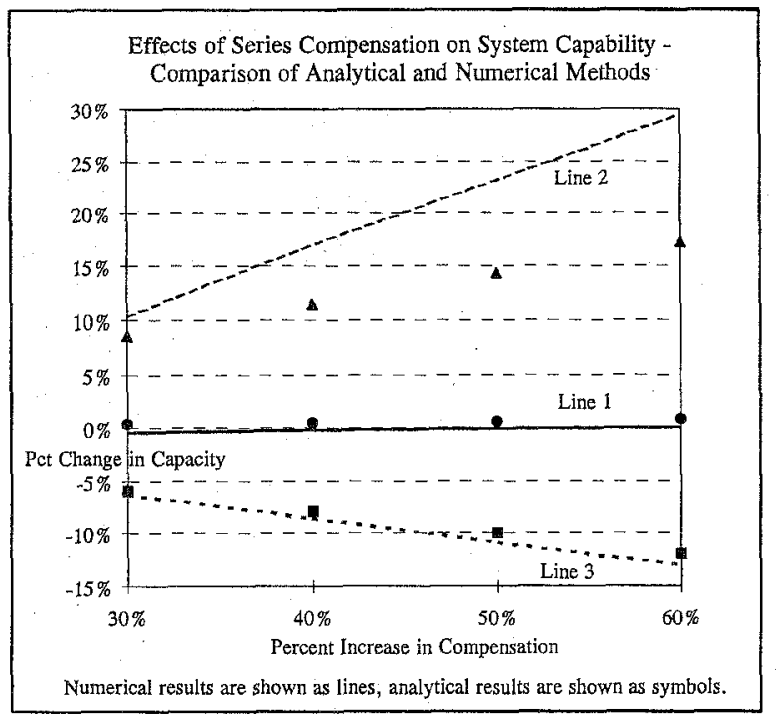

Fig. 5

Table 2 shows the analytical and simulated results of series compensation for a variety of the lines and for a selection of the compensation values examined.

Table 2 - Power Transfer Capacity - MW

\begin{tabular}{|cc|ccc|}
\hline \multirow{2}{*}{ Line Being Compensated } & \multicolumn{3}{|c|}{ Percent Compensation } \\
\cline { 3 - 5 } & & $20 \%$ & $40 \%$ & $60 \%$ \\
\hline Line 1: 1 to 4 & $\mathrm{A}$ & 238.7 & 228.8 & 218.9 \\
& $\mathrm{~N}$ & 236.2 & 227.3 & 216.1 \\
\hline Line 2: 1 to 6 & $\mathrm{A}$ & $\mathbf{2 6 2 . 7}$ & 276.8 & $\mathbf{2 9 1 . 0}$ \\
& $\mathrm{N}$ & $\mathbf{2 6 5 . 3}$ & $\mathbf{2 9 0 . 5}$ & $\mathbf{3 2 1 . 5}$ \\
\hline Line 3: 2 to 3 & $\mathrm{A}$ & 249.2 & 249.8 & 250.5 \\
& $\mathrm{~N}$ & 247.1 & 247.9 & 248.5 \\
\hline Line 4: 2 to 5 & $\mathrm{A}$ & 247.8 & 247.0 & 246.3 \\
& $\mathrm{~N}$ & 247.5 & 246.6 & 245.4 \\
\hline Line 6: 4 to 6 & $\mathrm{A}$ & 246.6 & 244.7 & 242.9 \\
& $\mathrm{~N}$ & 246.4 & 242.2 & 240.5 \\
\hline Line 7: 5 to 6 & $\mathrm{A}$ & 248.9 & 249.3 & 249.8 \\
& $\mathrm{~N}$ & 248.9 & 247.3 & 250.0 \\
\hline
\end{tabular}

A denotes the analytical solution, and $\mathrm{N}$ denotes the simulated solution; Boldface denotes cases where the two outcomes differ by more than $2 \%$.

\section{Conclusions}

This paper studies the interplay between generation dispatch choices and series compensation devices as they affect power transfer capability of a system. The paper presents an approach for identifying the congestion conditions that lead to the need for such series devices, and provides a means to quantify the benefits that such devices have on the system. A more comprehensive analysis can be performed by considering the benefits over a large number of likely operating conditions and dispatch scenarios, taking into consideration uncertainty that may exist in the future in the siting of generation facilities.

Transmission systems are not fixed entities. Series impedances can - indeed, will be - altered in ways that improve security margins and available transfer capability (maximum loadability) of power systems. Furthermore, there are direct tradeoffs between transmission capability and generation costs. However, decisions to invest in series devices that alter flows and capabilities will require justification by formal analysis. Such justification can only be provided by taking an integrated approach to the entire grid operation and quantifying the net benefits that such installations have on system margins and capabilities.

These margins and capabilities can be directly converted to economic benefits of all participants within power markets, and thus compared to the economic carrying costs on investment in compensation technologies within a cost-benefit framework.

\section{Endnote}

[1] The methodology, Marginal Costing Within Transmission Networks (MCTN), was originally developed by LRCA under the auspices of a joint Southern Company/EPRI research project. The MCTN methodology will be described in a soon-tobe-published EPRI Report and Technical Brief.

\section{Acknowledgment}

This paper does not necessarily represent the views of Laurits $R$. Christensen Associates or of Siemens. The authors remain solely responsible for any errors and omissions in this paper. 


\section{References}

[1] FERC Order 888: Final Rule, "Promoting wholesale competition through open access non-discriminatory transmission services by public utilities; recovery of stranded costs by public utilities and transmitting utilities", Docket Nos. RM95-8-000 and RM94-7-001, April 24, 1996.

[2] NERC Report, "Transmission reservation and scheduling", Transmission Reservation and Scheduling Task Force, Report to the Engg. Committee and Oper. Committee, Dec. 3-4, 1996.

[3] Alvarado F.L., Ray D., Kirsch L., and Stevenson R. The value of transmission security. Electric Power Research Institute, report RP 4000-14, Nov. 1993.

[4] Caves D., Herriges J., Windle R., "Customer demand for service reliability in the electric power industry: A synthesis of the outage cost literature", Bulletin of Economic Reseacrh, vol. 4.2, no. 2, pp. 79-119, 1990.

[5] Dobson I., L. Lu, "Computing an optimum direction in control space to avoid saddle node bifurcation and voltage collapse in electric power systems", IEEE Transactions on Automatic Control, vol 37, no. 10, October 1992, pp. 16161620.

[6] Dobson I. "Computing a closest bifurcation instability in rnultidimensional parameter space", J. of Nonlinear Science, vol. 3, pp. 307-327, 1993.

[7] Greene S., I. Dobson, F.L. Alvarado, "Sensitivity of the loading margin to voltage collapse with respect to arbitrary parameters", paper 96WM278-2-PWRS, IEEE PES Winter meeting, Baltimore, MD, January 1996 and to appear in IEEE Transactions on Power Systems.

[8] Greene S., "Margin and sensitivity methods for steady state stability analysis of power systems", Preliminary report for PhD, University of Wisconsin-Madison, December 1996.

[9] Van Cutsem T. "A method to compute reactive power margins with respect to voltage collapse", IEEE Trans. Power Systems, vol. 6, no. 1, pp. 145-156, Feb. 1991.

[10] Baldick R. and Kahn E., "Contract paths, phase-shifters, and efficient electricity trade", Paper 96 SM 495-2 PWRS, IEEE/PES Summer Meeting, Denver 1996.

[11] Canizares C.A. and Alvarado F.L., "Point of collapse and continuation methods for large AC/DC systems", IEEE Trans. Power Systems, vol. 8, no. 1, pp. 1-8, Feb. 1993.

[12] Bergen A.R. Power system analysis. Prentice Hall, New Jersey, 1986.

[13] Wood A.J., and Wollenberg B.F. Power generation, operation and control. Wiley, New York, 1984

\section{Appendix A}

This section informally derives formula 1 of the paper, and gives an analogous formula for the case when the line limits are constrained by MVA rating. The references [12], [13], [6] may be useful. Voltage collapse [6] is not assumed to occur.

Let the power system nodes be numbered $1,2, \ldots$, $\mathrm{M}, \mathrm{M}+1, \ldots, \mathrm{N}$, where the first $\mathrm{M}$ buses are generators; the remaining buses are load buses. Let the voltage and angle at bus $i$ be denoted by $\left|V_{i}\right|$ and $\theta_{i}$ respectively, with bus 1 being a reference bus: $\theta_{1}=0$. Denote the branch admittance between $\mathrm{i}$ and $\mathrm{k}$ as $\mathrm{y}_{\mathrm{ik}}=\mathrm{g}_{\mathrm{ik}}+\mathrm{j} \mathrm{b}_{\mathrm{ik}}$; denote the shunt admittance at bus $\mathrm{i}$ to be $\mathrm{y}_{\mathrm{ii}}=\mathrm{g}_{\mathrm{ii}}+\mathrm{jb}_{\mathrm{ii}}$. Denote the real power and reactive power flow from bus $i$ to bus $k$ to be $P_{i k}$ and $Q_{i k}$ respectively:

$P_{i k}=\left|V_{k}\right|^{2} g_{i k}-\left|V_{k}\right|\left|V_{i}\right|\left(g_{i k} \cos \left(\theta_{i}-\theta_{k}\right)+b_{i k} \sin \left(\theta_{i}-\theta_{k}\right)\right)$ $\mathrm{Q}_{\mathrm{ik}}=-\left|\mathrm{V}_{\mathbf{k}}\right|^{2} \mathrm{~b}_{\mathrm{ik}}-\left|\mathrm{V}_{\mathbf{k}}\right|\left|\mathrm{V}_{\mathrm{i}}\right|\left(\mathrm{g}_{\mathrm{ik}} \sin \left(\theta_{\mathrm{i}}-\theta_{\mathrm{k}}\right)-\mathrm{b}_{\mathrm{ik}} \cos \left(\theta_{\mathrm{i}}-\theta_{\mathrm{k}}\right)\right)$

Denote $\mathbf{P}_{G}=\left[P_{1}, P_{2}, \ldots, P_{M}\right]^{t}$, a vector of generator outputs; $\quad \mathbf{P}_{\mathbf{L}}=\left[-\mathbf{P}_{\mathrm{M}+1}, \quad-\mathbf{P}_{\mathrm{M}+2}, \ldots,-\mathbf{P}_{\mathrm{N}}\right], \quad \mathbf{Q}_{\mathbf{L}}=\left[-\mathbf{Q}_{\mathrm{M}+1}, \quad-\right.$ $\left.\mathbf{Q}_{\mathrm{M}+2}, \ldots,-\mathrm{Q}_{\mathrm{N}}\right]$ are vectors of real power and reactive power loads respectively. Let $\theta=\left[\theta_{2}, \theta_{3}, \ldots, \theta_{N}\right]^{t}$, and $\mathrm{V}=\left[\left|\mathrm{V}_{\mathrm{M}+1}\right|,\left|\mathrm{V}_{\mathrm{M}+2}\right|, \ldots,\left|\mathrm{V}_{\mathrm{N}}\right|\right]^{\mathrm{t}}$.

Let $\mathbf{k}_{\mathrm{PL}}, \mathbf{k}_{\mathrm{QL}}$ denote the real power and reactive power participation factors of the load buses in a load growth scenario. Let $\mathbf{k}_{\mathbf{G}}$ denote a vector of length $\mathbf{M}$ representing the participation factors in a distributed slack generation dispatch. To eliminate the extraneous distributed slack equation, we define a matrix $\mathbf{L}_{\mathbf{G}}$ of dimension (M-1) $\mathrm{xM}$ whose rows form a basis for the space orthogonal to $\mathbf{k}_{\mathrm{G}}$, i.e., $\mathbf{L}_{\mathrm{G}} \mathbf{k}_{\mathrm{G}}=\mathbf{0}$ (for the case of $\mathbf{a}$ single slack bus, the operation $\mathbf{L}_{G} \mathbf{P}_{G}$ is equivalent to dropping the slack bus real power equation). A load growth scenario is described by the following complete set of equations:

$$
\left(\begin{array}{c}
\mathbf{L}_{\mathbf{G}} \mathbf{P}_{\mathbf{G}} \\
-\mathbf{P}_{\mathbf{L}} \\
-\mathbf{Q}_{\mathbf{L}}
\end{array}\right)=\left(\begin{array}{c}
\mathbf{L}_{\mathbf{G}} \mathbf{P}_{\mathbf{G} 0} \\
-\mathbf{P}_{\mathbf{L} 0} \\
-\mathbf{Q}_{\mathbf{L} 0}
\end{array}\right)+\left(\begin{array}{c}
\mathbf{L}_{\mathbf{G}} \mathbf{k}_{\mathbf{G}} \beta \\
-\mathbf{k}_{\mathbf{P L}} \alpha \\
-\mathbf{k}_{\mathbf{Q L}} \alpha
\end{array}\right)=\left(\begin{array}{c}
\mathbf{L}_{\mathbf{G}} \mathbf{P}_{\mathbf{G} 0} \\
-\mathbf{P}_{\mathbf{L} 0} \\
-\mathbf{Q}_{\mathbf{L} 0}
\end{array}\right)+\left(\begin{array}{c}
0 \\
-\mathbf{k}_{\mathbf{P L}} \alpha \\
-\mathbf{k}_{\mathrm{QL}} \alpha
\end{array}\right)
$$

where $\alpha$ is a scalar that measures the load growth amount and $\beta$ is the corresponding increase in system generation output, and the zero in the subscripts refers to the base case. At a certain $\alpha$, cascading overloads will occur on point $*$ on the OLB (Figure 2) initiated by a flow limit on a critical line from bus $m$ to bus $n$.

Case 1: MW flow limit. We want to estimate the change in $\alpha$ if $\Delta t \%$ series compensation is added in the line between $r$ and $s$ (lines $m-n, r-s$ are different). We first linearize the load flow equations A1 at OLB point * $(\Delta$ denotes changes):

(A2) $\left(\begin{array}{c}0 \\ -\mathbf{k}_{\mathrm{PL}} \Delta \alpha \\ -\mathbf{k}_{\mathrm{QL}} \Delta \alpha\end{array}\right)=\mathbf{J}_{\mathrm{G}}\left(\begin{array}{c}\Delta \theta \\ \Delta V\end{array}\right)+\mathbf{S}_{\mathrm{G}} \mathbf{B}_{\mathrm{ser}} \Delta \mathrm{b}_{\mathrm{rs}}+\mathbf{S}_{\mathrm{G}} \mathbf{C}_{\mathrm{ser}} \Delta \mathrm{g}_{\mathrm{rs}}$

where $\mathbf{J}_{\mathbf{G}}$ is the Jacobian of size (2N-M-1) $x(2 \mathrm{~N}-\mathrm{M}-1)$, $S_{G}=\left[\begin{array}{cc}\mathbf{L}_{G} & 0 \\ \mathbf{0} & \mathbf{I}_{2 \mathrm{~N}-\mathrm{M}}\end{array}\right]$ and $\mathbf{B}_{\mathrm{ser}}$ and $\mathbf{C}_{\mathrm{ser}}$ are column vectors: 


$$
\mathbf{B}_{\text {ser }}(i)=\left\{\begin{array}{cc}
-\left|V_{r}\right| \mid V_{s} s \sin \left(\theta_{r}-\theta_{s}\right) & i=r-1 \neq 0 \\
-\left|V_{r}\right|\left|V_{s}\right| \sin \left(\theta_{s}-\theta_{r}\right) & i=s-1 \neq 0 \\
-\left|V_{r}\right|^{2}+\left|V_{r}\right|\left|V_{s}\right| \cos \left(\theta_{s}-\theta_{r}\right) & i=r+M-1>2 M-1 \\
-\left|V_{s}\right|^{2}+\left|V_{r}\right|\left|V_{s}\right| \cos \left(\theta_{s}-\theta_{r}\right) & i=s+M-1>2 M-1 \\
0 & \text { otherwise }
\end{array}\right.
$$

All voltages and angles are evaluated at the OLB point *.

Next, linearize the $P_{\text {ma }}$ flow equation at OLB point *: (A3) $\quad \Delta \mathrm{P}_{\mathrm{mn}}=\mathrm{D}\left(\begin{array}{c}\Delta \theta \\ \Delta \mathrm{V}\end{array}\right)$

where $\mathbf{D}$ is row vector of size $2 \mathrm{~N}-\mathrm{M}-1$ with $\mathrm{i}^{\text {th }}$ element:

$$
\left\{\begin{array}{cc}
\left|V_{m}\right|\left|V_{n}\right|\left(g_{m i} \sin \left(\theta_{m}-\theta_{n}\right)-b_{m m} \cos \left(\theta_{m}-\theta_{n}\right)\right) & i=m-1 \neq 0 \\
\left|V_{m}\right|\left|V_{n}\right|\left(-g_{m m} \sin \left(\theta_{m}-\theta_{n}\right)+b_{m n} \cos \left(\theta_{m}-\theta_{n}\right)\right) & i=n-1 \neq 0 \\
2\left|V_{m}\right| g_{m}-\left|V_{n}\right|\left(g_{m} \cos \left(\theta_{m}-\theta_{n}\right)-b_{m m} \sin \left(\theta_{m}-\theta_{n}\right)\right) & i=M+m-1>2 M-1 \\
-\left|V_{m}\right|\left(g_{m n} \cos \left(\theta_{m}-\theta_{n}\right)-b_{m n} \sin \left(\theta_{m}-\theta_{n}\right)\right) & i=M+n-1>2 M-1 \\
0 & \text { otherwise }
\end{array}\right.
$$

Eliminate the angles and voltages from (A2) and (A3):

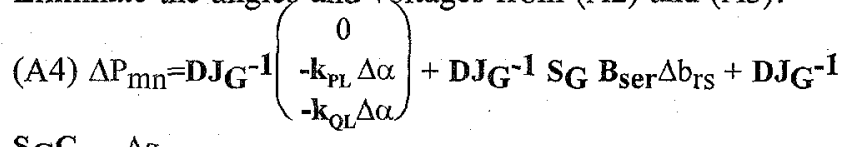

$\mathrm{S}_{\mathbf{G}} \mathrm{C}_{\text {ser }} \Delta \mathrm{g}_{\mathrm{rs}}$

Since $P_{m n}$ is already at its flow limit, it cannot increase: $\Delta P_{m n} \leq 0$. Thus, the maximum $\Delta \alpha$ subject to this constraint is given by setting $\Delta \mathrm{P}_{\operatorname{mn}}=0$ in (A4). For a $\Delta t \%$ series compensation, it can be shown that:

$$
\Delta \alpha=\Lambda_{1 \mathrm{G}} \frac{\partial \mathrm{b}_{r s}}{\partial \mathrm{t}} \Delta \mathrm{t}+\Lambda_{2 \mathrm{G}} \frac{\partial \mathrm{g}_{r s}}{\partial \mathrm{t}} \Delta \mathrm{t}
$$

where:

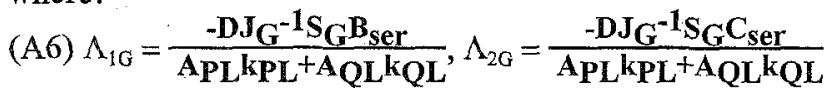

$$
\begin{aligned}
& \text { (A7) } \frac{\partial \mathrm{b}_{\mathrm{rs}}}{\partial \mathrm{t}}=\frac{\mathrm{b}_{\mathrm{rs}}\left(\mathrm{b}_{\mathrm{rs}}{ }^{2}-\mathrm{g}_{\mathrm{s}}{ }^{2}\right)}{100\left(\mathrm{~g}_{\mathrm{rs}}{ }^{2}+\mathrm{b}_{\mathrm{rs}}{ }^{2}\right)} ; \frac{\partial \mathrm{g}_{\mathrm{rs}}}{\partial \mathrm{t}}=\frac{2 \mathrm{~g}_{\mathrm{rs}} \mathrm{b}_{\mathrm{rs}}{ }^{2}}{100\left(\mathrm{~g}_{\mathrm{rs}}{ }^{2}+\mathrm{b}_{\mathrm{rs}}{ }^{2}\right)}
\end{aligned}
$$

and $\mathbf{A}_{\mathrm{PL}}, \mathbf{A}_{\mathrm{QL}}$ are row vectors containing elements $\mathrm{M}$ through $\mathrm{N}-1$, and elements $N$ through $2 \mathrm{~N}-\mathrm{M}-1$, respectively, of the row vector $\mathbf{D J}_{\mathbf{G}}^{-1}$.

Case 2: MVA flow limit. For an MVA flow limit (instead of the MW flow limit) in line $n-m$, the analogue of formula (A5) is obtained similarly. Let $\beta_{1} \frac{P_{m n}}{\sqrt{\mathrm{P}^{2}{ }_{m n}+Q^{2}}}$ , $\beta_{2}=\sqrt{1-\beta_{1}^{2}}$. Replace $\mathbf{D}$ in formula (A6) with $\mathbf{D}_{\mathrm{MVA}}=$ $\beta_{1} \mathbf{D}+\beta_{2} \mathbf{D}_{\mathrm{Q}}$, where $\mathbf{D}_{\mathbf{Q}}(\mathrm{i})=$

$$
\left\{\begin{array}{cc}
\left|V_{m}\right|\left|V_{n}\right|\left(-g_{m n} \cos \left(\theta_{m}-\theta_{n}\right)-b_{m n} \sin \left(\theta_{m}-\theta_{n}\right)\right) & i=m-1 \neq 0 \\
\left|V_{m}\right|\left|V_{n}\right|\left(-g_{m n} \cos \left(\theta_{m}-\theta_{n}\right)+b_{m n} \sin \left(\theta_{m}-\theta_{n}\right)\right) & i=n-1 \neq 0 \\
2\left|V_{m}\right| b_{m n}-\left|V_{n}\right|\left(g_{m n} \sin \left(\theta_{m}-\theta_{n}\right)-b_{m n} \cos \left(\theta_{m}-\theta_{n}\right)\right) & i=M+m-1>2 M-1 \\
-\left|V_{m}\right|\left(g_{m n} \sin \left(\theta_{m}-\theta_{n}\right)-b_{m n} \cos \left(\theta_{m}-\theta_{n}\right)\right) & i=M+n-1>2 M-1 \\
0 & \text { otherwise. }
\end{array}\right.
$$

This completes the equations.

\section{Biographies}

Rajesh Rajaraman. Dr. Rajaraman is a senior engineer with Christensen Associates. He is currently involved in spot pricing, transmission pricing and planning, and ancillary services pricing at the wholesale and retail level. He holds a Ph.D. from the University of Wisconsin-Madison.

Fernando Alvarado. Dr. Alvarado is a leader in the field of computer modeling of electric system networks and has developed a state-of-the-art methodology based upon Point-ofCollapse Loadflow. He earned a Ph.D. in Computer, Information and Control (EE) from Michigan in 1972 and is currently a Professor of Electrical and Computer Engineering at the University of Wisconsin.

Arthur Maniaci. Mr. Maniaci, a senior engineer at Christensen Associates, specializes in statistical and engineering analyses of electricity utilization, including transmission systems. He has extensive experience in the electric utility industry, primarily in supply- and demand-side planning. He has published several papers for the A.E.I.C. and holds an M.S.M.E. from UWMadison.

Robert Camfield. Mr. Camfield is a senior economist at Christensen Associates with extensive experience in the electric power industry, most recently at The Southern Company. He managed a major overseas consulting project in Ankara, Turkey for Southern Electric International, focusing on economic costing and pricing. Mr. Camfield was also a chief economist of the New Hampshire PUC. He is a graduate of Interlochen Arts Academy and holds a M.A. in economics from Western Michigan University.

Sasan Jalali. Dr. Jalali is employed with Siemens Energy and Automation, Atlanta. He is involved in applications of FACTS devices, power quality, and transmission planning. Dr. Jalali received his Ph.D. at the University of Wisconsin-Madison. 